\title{
Proposed continuous wave energy recovery operation of an x-ray free electron laser
}

\author{
J. Sekutowicz, S. A. Bogacz, D. Douglas, P. Kneisel, and G. P. Williams \\ Thomas Jefferson National Accelerator Facility (JLab), 12000 Jefferson Avenue, Newport News, Virginia 23606, USA
}

M. Ferrario

Istituto Nazionale di Fisica Nucleare, Via E. Fermi 40, 00044 Frascati, Italy

I. Ben-Zvi, J. Rose, J Smedley, and T. Srinivasan-Rao

Brookhaven National Laboratory, Upton, New York 11973, USA

L. Serafini

Istituto Nazionale di Fisica Nucleare, Via Celoria 16, 20133 Milan, Italy

W.-D. Möller, B. Petersen, D. Proch, and S. Simrock

Deutsches Elektronen-Synchrotron, Notkestrasse 85, 22603 Hamburg, Germany

P. Colestock

Los Alamos National Laboratory, Los Alamos, New Mexico 87545, USA

J. B. Rosenzweig

University of California Los Angeles, 405 Hilgard Avenue, Los Angeles, California 90095, USA

(Received 16 December 2003; published 10 January 2005)

\begin{abstract}
Commissioning of two large coherent light facilities (XFELs) at SLAC and DESY should begin in 2008 and 2011, respectively. In this paper we look further into the future, hoping to answer, in a very preliminary way, two questions. First: What will the next generation of XFEL facilities look like? Believing that superconducting technology offers advantages such as high quality beams with highly populated bunches, the possibility of energy recovery and higher overall efficiency than warm technology, we focus this preliminary study on the superconducting option. From this belief the second question arises: What modifications in superconducting technology and in the machine design are needed, as compared to the present DESY XFEL, and what kind of research and development program should be proposed to arrive in the next few years at a technically feasible solution with even higher brilliance and increased overall conversion of ac power to photon beam power? In this paper we will very often refer to and profit from the DESY XFEL design, acknowledging its many technically innovative solutions.
\end{abstract}

DOI: 10.1103/PhysRevSTAB.8.010701

PACS numbers: 29.27.-a, 29.17.+w

\section{INTRODUCTION}

Two high brilliance pulsed large coherent light facilities (XFELs) have been proposed: one at SLAC [1] and another one at DESY [2] (by the European collaboration). The maximum expected peak brilliances ${ }^{1}$ at the shortest wavelength of about $1 \AA$ are $8.5 \times 10^{32}$ and $5.4 \times 10^{33}$ for the SLAC and DESY facilities, respectively. The machines differ in the expected average brilliance. The DESY XFEL will provide average brilliance of $1.6 \times 10^{25}$ (roughly 380 times higher than the facility at SLAC) as a result of different driving linac technologies used in each project. The driving accelerator for the Linac Coherent Light Source (LCLS) at SLAC is based on copper accelerating structures operating at room temperature, while the accelerator for the XFEL at DESY uses superconducting niobium cavities operating at $2 \mathrm{~K}$. However, the single

\footnotetext{
${ }^{1}$ Units: photon $/ \mathrm{s} / \mathrm{mm}^{2} / \mathrm{mrd}^{2} / 0.1 \%$ bandwidth.
}

bunch parameters of both designs are quite similar. The maximum final beam energy is $15 \mathrm{GeV}$ in the case of the LCLS and $20 \mathrm{GeV}$ in the case of the DESY XFEL. For both, the charge per bunch is $1 \mathrm{nC}$ and the transverse normalized emittance is $\sim 1.4 \mu \mathrm{mrad}$. The $\mathrm{rms}$ bunch length is $80 \mathrm{fs}$ (DESY) and $97 \mathrm{fs}$ (LCLS). The difference in the average brilliance mostly results from the number of bunches per second, which is 120 in the LCLS design and 40000 in the DESY XFEL design. The superconducting linac of the DESY XFEL supports longer trains of bunches (up to 4000 bunches with a repetition frequency of $10 \mathrm{~Hz}$ ), even when operated at the maximum energy of $20 \mathrm{GeV}$.

Recent experimental results of $\mathrm{GeV}$ scale energy recovery in the superconducting CEBAF accelerator at the Thomas Jefferson National Accelerator Facility (JLab) [3] are quite encouraging. This combined with continuing progress in the performance of superconducting cavities, demonstrated at many laboratories, e.g., DESY [4] and JLab [5], initiated our discussions. 
Here we explore the feasibility of continuous wave (cw) or semi-cw (single long rf pulse per second) operational mode for a future XFEL facility. One recognizes that this mode will significantly increase the average brilliance of the XFEL. In the case of the DESY XFEL, a large fraction of each rf pulse (total pulse length $t_{p}=1.3 \mathrm{~ms}$ ) is needed to build up the $e-m$ fields in the superconducting cavities before they become useful for acceleration (buildup time $t_{r}=0.5 \mathrm{~ms}$ ). Pulsed rf systems (modulators and klystrons) usually do not allow one to increase significantly the beam on-time, so one has to look for an alternative solution. A 920 MW scale cw or semi-cw rf system (these numbers will be justified later) would prohibitively increase the overall cost of the facility. The only remedy known to us is to operate in the energy recovery (ER) mode.

\section{A. Energy recovery at JLab}

In 2002, in an effort to address the issues of energy recovering high-energy beams, Jefferson Lab proposed a minimally invasive energy recovery experiment utilizing $\mathrm{CEBAF}$ - the $6 \mathrm{GeV}$ recirculating superconducting accelerator [3]. The experiment was successfully carried out at the end of March 2003 by demonstrating energy recovery of a $1 \mathrm{GeV}$ beam. Until this experiment, there were no plans aimed at addressing issues related to beam quality preservation in systems with large final beam energies (up to $1 \mathrm{GeV}$ ) or large energy ratios between final and injected beams (a factor of 50). A schematic representation of CEBAF with the energy recovery experiment is illustrated in Fig. 1. Beam is injected into the North Linac (NL) at $20 \mathrm{MeV}$ where it is accelerated to $520 \mathrm{MeV}$. The beam traverses arc 1 and then begins acceleration through the South Linac (SL) where it reaches a maximum energy of $1020 \mathrm{MeV}$. Following the SL, the beam passes through a newly installed phase delay chicane. The chicane was designed to create a path length differential of exactly $1 / 2$ rf wavelength so that upon reentry into the NL the beam is $180^{\circ}$ out of phase with the cavities and will subsequently be decelerated to $520 \mathrm{MeV}$. After traversing arc 1 a second time the beam enters the SL-still out of phase with the cavities - and is decelerated to $20 \mathrm{MeV}$, at which point the spent electron beam is sent to a dump. In this way the beam gives energy back, which is used to

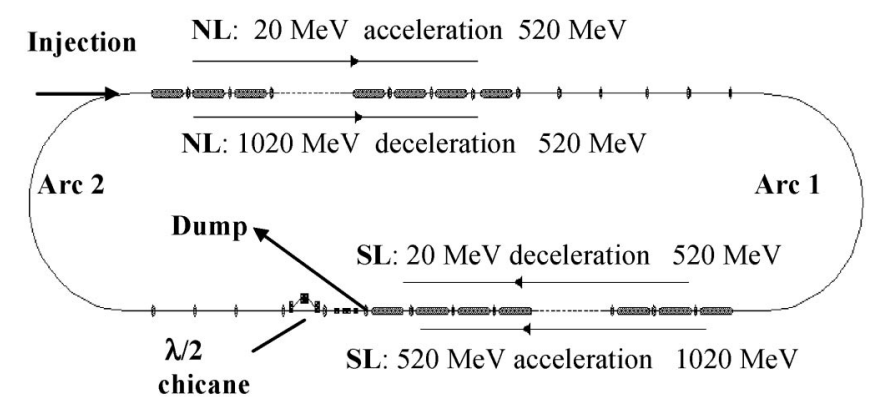

FIG. 1. CEBAF energy recovery experiment at JLab. accelerate subsequent beam. Even the problem of finding a beam transport setting that was a compromise between the widely differing energies of the accelerated and decelerated beam was solved. The conclusion drawn from this experiment is that there is no limitation preventing energy recovery at higher beam energies. Furthermore, measured beam quality characteristics showed no degradation of the initial phase space during the energy recovery process. The beam profile was consistent with a Gaussian distribution down to 5 orders of magnitude in intensity. Later, we will propose an energy recovery option based on the injection of the decelerated beam backwards from the high-energy side, which completely eliminates potential problems with optics matching.

\section{B. Cryoplant capacity versus pulse length}

Because of cavity dynamic wall losses and long integrated rf on-time, the $\mathrm{cw}$ and semi-cw operational modes will require a bigger cryogenic plant than the pulsed option. The cryogenic plant of the DESY XFEL (with a duty cycle of only $1 \%$ ) will have $1.5 \mathrm{~kW}$ capacity at $2 \mathrm{~K}$ (with $\sim 50 \%$ overhead). To keep a reasonable size of the cryoplant for the cw XFEL three following modifications shall be made: (i) increase linac length by $\sim 16 \%$ (compared to the present DESY XFEL design), (ii) operate cavities at $E_{\text {acc }}$ up to $15 \mathrm{MV} / \mathrm{m}$ (nominal operation) and up to $\sim 22 \mathrm{MV} / \mathrm{m}$ (extended operation), and (iii) operate the linac in cw or semi-cw mode with adjustable duty cycle versus $E_{\text {acc }}$ to stay within available cryogenic budget.

The chosen nominal gradient ensures high intrinsic quality factor $Q_{o}$ of the superconducting cavities. Recent experience with $1.3 \mathrm{GHz}$ TTF (TESLA Test Facility) cavities at DESY shows that at $2 \mathrm{~K}$ these bulk $\mathrm{Nb}$ cavities can be operated at $E_{\text {acc }}$ up to $20 \mathrm{MV} / \mathrm{m}$ without $Q_{o}$ degradation. The confirming example in Fig. 2 shows 14 TTF cavities (third production) with high $Q_{o}=1.5-2.1 \times$ $10^{10}$ at $15 \mathrm{MV} / \mathrm{m}$ (the BCS $Q_{o}$ at $2 \mathrm{~K}$ is $2.3 \times 10^{10}$ ). One can possibly reduce the residual surface resistance by improved preparation methods. In the next sections

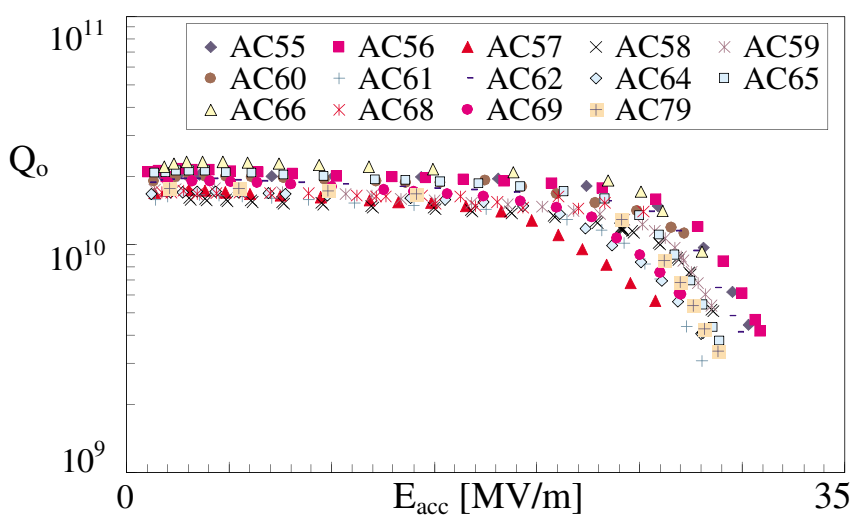

FIG. 2. (Color) $Q_{o}$ vs $E_{\text {acc }}$ of $14 \mathrm{TTF}$ cavities from the third production at DESY. 


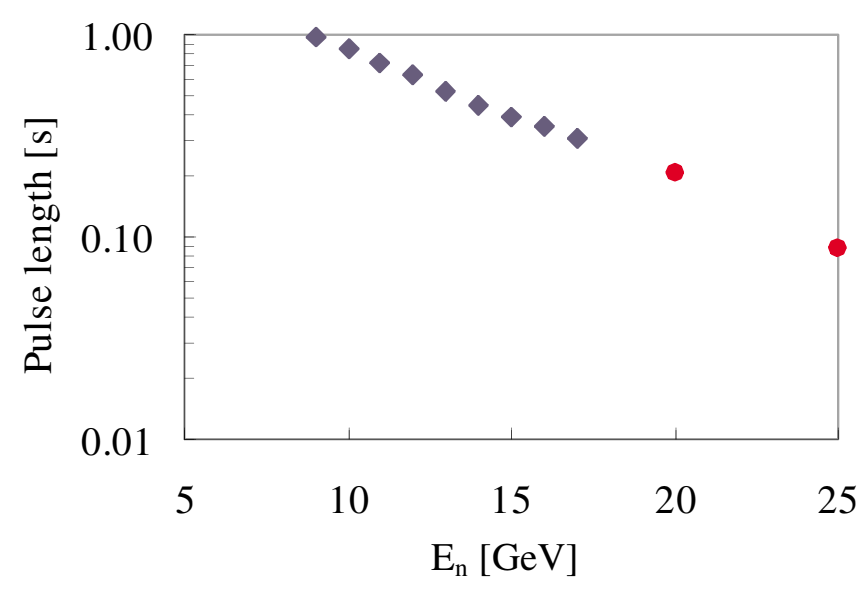

FIG. 3. (Color) rf pulse length versus final beam energy $E_{n}$. Nominal range (diamonds), extended range (solid circles).

we will assume that on average $Q_{o}$ is $1.7 \times 10^{10}$ at $15 \mathrm{MV} / \mathrm{m}$, dropping to $1.3 \times 10^{10}$ at $22 \mathrm{MV} / \mathrm{m}$. In the $\mathrm{cw}$ operational mode the dynamic loss per cell at $E_{\text {acc }}=$ $15 \mathrm{MV} / \mathrm{m}$ is $1.65 \mathrm{~W}$. An active linac length of $1132 \mathrm{~m}$ is needed to reach energy of $17 \mathrm{GeV}$. The dynamic loss due to the fundamental mode for the whole linac at $2 \mathrm{~K}$ would be $16 \mathrm{~kW}$. The above cryogenic load can be significantly lowered if the linac is operated in a semi-cw mode, with a single, long pulse each second. The length of the pulse can be scaled with $\sim\left(E_{\text {acc }}\right)^{-2}$, while the $1 \mathrm{~Hz}$ repetition frequency is kept constant. If experiments require lower energy photons the linac can be operated at lower gradients and the pulse length can be increased. All the above considerations lead to the requirement for a cryoplant with $8.5 \mathrm{~kW}$ capacity at $2 \mathrm{~K}$ (including $\sim 40 \%$ overhead). This choice is rather arbitrary since the cryoplant capacity can be optimized with respect to a variety of parameters, such as the linac length, the operating temperature, the rf pulse length, and capital cost. The chosen cryoplant size is $1 / 3$ of the cryoplant proposed for the TESLA collider [6] and is of the order of the cryoplant at JLab $(5 \mathrm{~kW})$. Figure 3 shows the maximum beam on-time versus the final beam energy $E_{n}$ when $2 / 3$ of the cryoplant capacity is used. Within the above energy range, the beam on-time varies from $1 \mathrm{~s}(\mathrm{cw})$ up to $E_{n}=9 \mathrm{GeV}$, to $206 \mathrm{~ms}$ at $E_{n}=20 \mathrm{GeV}$, and to $88 \mathrm{~ms}$ at $E_{n}=25 \mathrm{GeV}$. The beam on-time is longer by a factor of 120 at $9 \mathrm{GeV}$ and by a factor of 26 at $20 \mathrm{GeV}$ than for the pulsed XFEL at DESY.

\section{LINAC}

A possible layout of the proposed $\mathrm{cw}$ facility is quite similar to the present elaborate layout of the DESY XFEL. Several components will make the $\mathrm{cw}$ facility different from the pulsed version. First of all, the $\mathrm{cw}$ facility requires a novel design of the rf gun, which can be operated in a cw mode. Present state-of-the art rf guns do not fulfill specifications of the device needed for $\mathrm{cw}$ XFEL. Vigorous

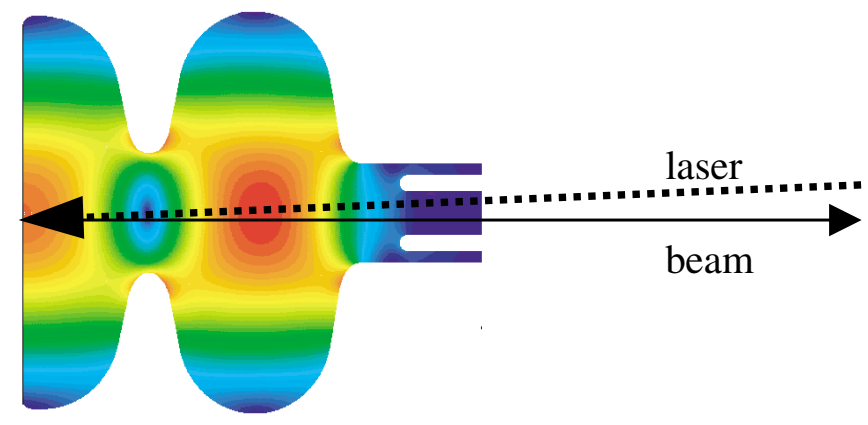

FIG. 4. (Color) Electric field contour in $1.3 \mathrm{GHz} 1.6$-cell $\mathrm{Nb}$ cavity with coaxial input coupler. Laser beam illuminates the back wall.

research and development (R\&D) programs [7], based on various approaches, at BNL, FZ-Rossendorf, Cornell University/JLab, Beijing University, and at AES/JLab generate optimism that a low emittance $(1 \mu \mathrm{m} \mathrm{rad}, \mathrm{rms})$, $\sim 1 \mathrm{nC}$, cw rf gun with a bunch frequency up to a few $\mathrm{MHz}$ will be available in the near future. We comment in the next subsection on the BNL solution. Another modification is the novel beam transport solution - a "teardrop" return arc combined with bypass minichicanes needed to avoid beam-beam interaction. The rf system itself will be described later.

\section{A. rf gun and injector}

As was already mentioned, we assume that the low emittance $\mathrm{cw}$ beam is generated by a rf gun. A very attractive approach has been proposed at BNL [8]. The basic idea is to illuminate the back wall of the superconducting $\mathrm{Nb}$ cavity with a UV laser. The niobium performs as the photocathode generating electrons which are accelerated by the rf field of the cavity. The 1.6 cell $\mathrm{Nb}$ cavity used here to design the injector is shown in Fig. 4. The $\mathrm{R} \& \mathrm{D}$ program at BNL is primarily focused on increasing the rather low intrinsic quantum efficiency $(\eta)$ of niobium. As was shown experimentally, various treatments of the surface, such as mechanical diamond polishing or/and laser cleaning, can increase $\eta$ from $2 \times 10^{-7}$ to $5 \times$ $10^{-5}$. A further increase of $\eta$ is possible when the emitting spot is exposed to a high electric field $E$. Recently, a single cell cavity at JLab was measured up to $E=88 \mathrm{MV} / \mathrm{m}$ of the wall peak field [9]. As confirmed by numerous acceptance tests of multicell cavities at DESY, the peak wall fields in the range $E=60-70 \mathrm{MV} / \mathrm{m}$ can be reached in $\mathrm{cw}$ operation without degradation of the cavity performance. Since $\eta$ scales linearly with the electric field ${ }^{2}$ applied at the emitting spot, one may expect that for $E=60-70 \mathrm{MV} / \mathrm{m}$, $\eta$ will increase to $10^{-4}$ or even above that value if higher energy photons can be used to illuminate the $\mathrm{Nb}$ wall. Figure 5 shows $\eta$ computed for two different photon en-

$$
{ }^{2} \eta=A(\nu)\left[h \nu-W+\sqrt{\left(e \cdot E / 4 \pi \varepsilon_{o}\right)}\right]^{2} .
$$




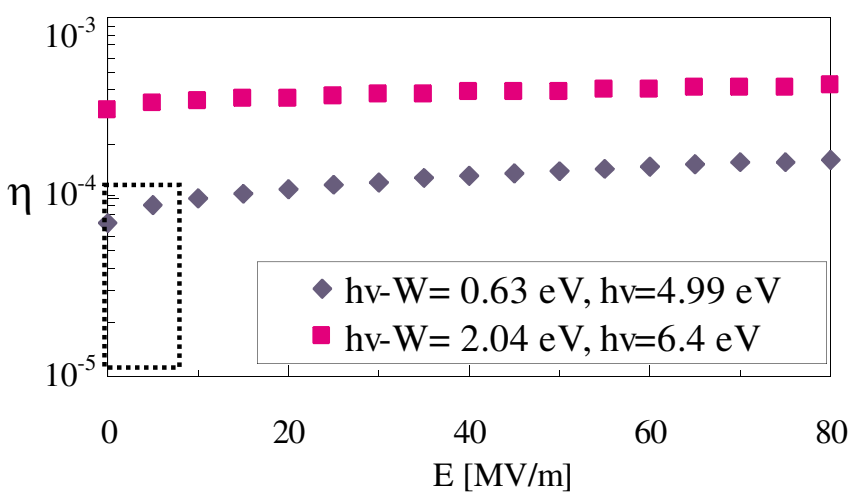

FIG. 5. (Color) Extrapolated change of $\eta$ versus applied electric field on the emitting spot. The curves are extrapolated for two photon energies $(h \nu)$. The marked area indicates dc measured data at BNL.

ergies, assuming a niobium work function of $W=4.4 \mathrm{eV}$. Any improvement in $\eta$ is of great importance. Even with $\eta=10^{-4}$, the laser power deposited in the $\mathrm{Nb}$ wall needed to generate a $\mathrm{cw}$ beam of several $\mathrm{MHz}$ and $1 \mathrm{nC}$ per bunch would probably be too high to keep the illuminated spot superconducting [10]. Fortunately, the emitting spot is exposed to a very low magnetic field and it is not obvious that a quench will occur. An experiment will be needed to find the maximum tolerable laser power. In addition, improving $\eta$ is important because the illuminating laser $^{3}$ itself will be technically very challenging. The proposed injector layout ${ }^{4}$ is shown in Fig. 6. In this $15 \mathrm{~m}$ long split injector, bunches are accelerated up to $120 \mathrm{MeV}$. Beam acceleration (gun cavity and eight 9-cell superconducting cavities) and beam focusing (solenoid) take place at different longitudinal positions. The layout opens a new possibility for a high brightness superconducting rf gun [13]. The beam and the injector parameters are listed in Table I.

\section{B. General layout of the "single arc" option}

The "single arc" scenario is shown in Fig. 7. After bunch shortening in the first bunch compressor (BC I) (from $\sigma_{z}=1.7 \mathrm{~mm}$ to $\sigma_{z}=0.25 \mathrm{~mm}, \mathrm{rms}$ ), bunches are accelerated to $0.5 \mathrm{GeV}$ and compressed again in BC II to $\sigma_{z}=0.1 \mathrm{~mm}$. Both compressors are positioned almost as in the original DESY XFEL linac design and thus they can be directly adopted. The compression to $\sigma_{z}=0.025 \mathrm{~mm}$ rms in BC III takes place after the acceleration to $2 \mathrm{GeV}$. This compressor needs to be modified (compared to the original DESY design) to provide separation of the accelerated and the decelerated beams. This can be accomplished by adding two extra dipole magnets (see Fig. 8) and by the proper positioning of the compressor.

\footnotetext{
${ }^{3} 44 \mathrm{~W}$ of laser power is needed when $\eta=10^{-4}, q=1 \mathrm{nC}$, $f=1 \mathrm{MHz}$, and $h \nu=4.4 \mathrm{eV}$.

${ }^{4}$ The injector and the emittance compensation scheme are discussed in detail in $[11,12]$.
}

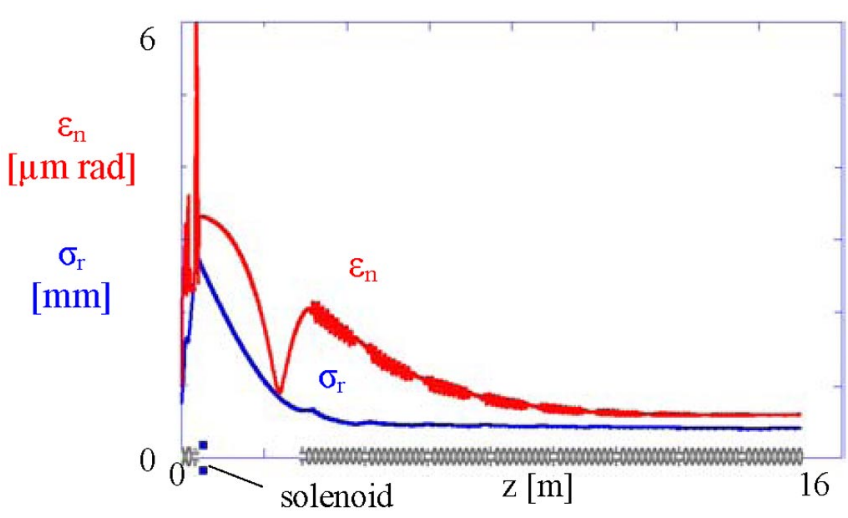

FIG. 6. (Color) Layout and beam functions of the split injector (acceleration and focusing take place at different longitudinal positions).

TABLE I. Parameters of the beam and the injector.

\begin{tabular}{lcc}
\hline \hline \multicolumn{1}{c}{ Parameter } & Units & \\
\hline rf-gun cavity operating frequency & $\mathrm{MHz}$ & 1300 \\
$E$ wall in rf-gun cavity & $\mathrm{MV} / \mathrm{m}$ & 60 \\
Emitting spot radius & $\mathrm{mm}$ & 1.5 \\
Charge & $\mathrm{nC}$ & 1 \\
Energy at the end of rf-gun & $\mathrm{MeV}$ & 6.5 \\
Normalized emittance at the cathode & $\mu \mathrm{m} \mathrm{rad}$ & 0.45 \\
$B$ field in the solenoid & $\mathrm{T}$ & 0.3 \\
Beam energy at the exit of the injector & $\mathrm{MeV}$ & 120 \\
$E_{\text {acc }}$ in eight superconducting cavities & $\mathrm{MV} / \mathrm{m}$ & 13.6 \\
Peak current & $\mathrm{A}$ & 50 \\
Bunch duration (flattop part) & $\mathrm{ps}$ & 20 \\
Transversal size $\sigma_{r}$ at the exit & $\mathrm{mm}$ & 0.5 \\
Normalized emittance $\varepsilon_{n}$ & $\mu \mathrm{m} \mathrm{rad}$ & 0.6 \\
at the exit of the injector & & \\
\hline \hline
\end{tabular}

The injector and the first part of the accelerator, up to $0.5 \mathrm{GeV}$ beam energy, will not be included into the ER "loop." The argument is that the decelerated beam would make an additional contribution to undesired transverse higher order modes (HOMs) already excited by the accelerated beam, which would in turn contribute to emittance degradation of the still low energy accelerated beam $(<$ $0.5 \mathrm{GeV}$ ). This effect will be studied more quantitatively in due course. Assuming an average beam current of $1 \mathrm{~mA}$, $0.5 \mathrm{MW}$ of $\mathrm{rf}$ power is needed for the operation of this part of the facility. Fortunately, 0.5 and $1.2 \mathrm{MW}$ rf sources are available at present on the market and no R\&D activity is needed.

The second part of the linac, above $0.5 \mathrm{GeV}$, has to be operated in the ER mode to lower the rf power needed for the operation. All bunches, after passing through optical devices, are turned around via a $180^{\circ}\left(270^{\circ}-90^{\circ}\right)$ teardrop loop and are injected back into the linac on the high-energy side. They are decelerated along the linac until the energy drops to $0.5 \mathrm{GeV}$. Finally, the decelerated beam is sent to a 


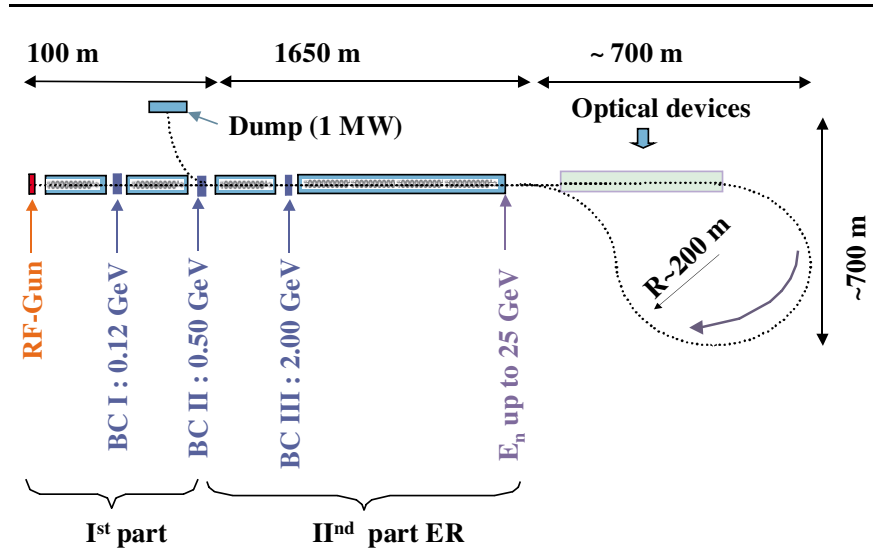

FIG. 7. (Color) Preliminary layout of the facility based on the single return arc.

dump. Deceleration takes place at the same gradient as acceleration. The main advantage of the injection from the high-energy side is that both the accelerated and decelerated beams maintain the same energy profile along the linac, which simplifies the optics design and guarantees uniform periodic focusing for both beams.

\section{Time structure of the beam}

Having two beams passing through the second part of the linac in opposite directions one has to avoid beambeam interaction, which may lead to beam quality degradation for the accelerated beam [14]. Keeping this in mind we propose the following three beam time structures.

\section{Nominal beam}

The nominal beam will consist of $1 \mathrm{nC}$ bunches with repetition frequency of $1 \mathrm{MHz}$ (time between two consecutive bunches $t_{b}=1 \mu \mathrm{s}$ ). The ER part of the linac is about $1650 \mathrm{~m}$ long, so only six accelerated bunches and six decelerated bunches will be in the ER part at the same time. The bunches of both beams will meet at 12 locations (see Fig. 9). Off-axis deflection (of a few millimeters) applied at these locations will be enough to suppress the beam-beam interaction to a negligible level. A single dipole magnet will bend the trajectories of the two counterpropagating beams (the accelerated and decelerated

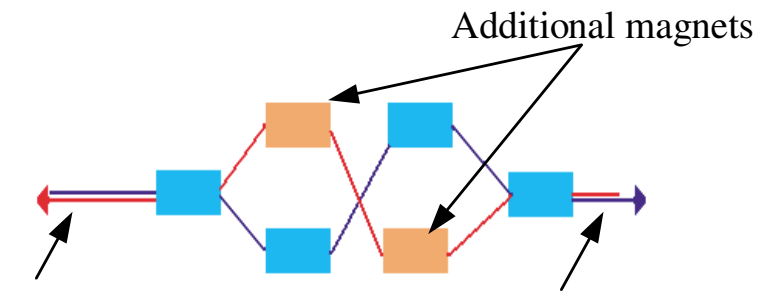

Decelerated beam

Accelerated beam

FIG. 8. (Color) Schematic layout of the modified bunch compressor BC III of DESY design to provide beam separation.

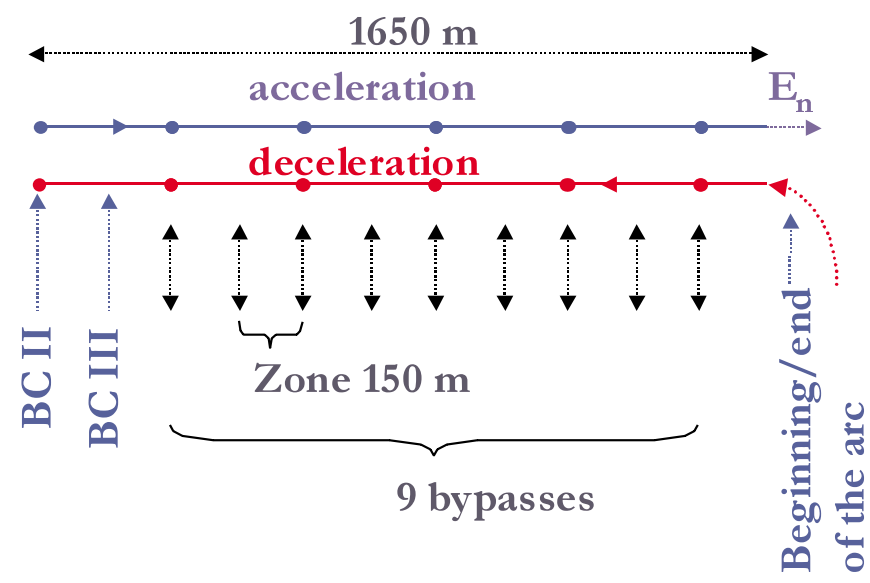

FIG. 9. (Color) Locations in which the accelerated and the decelerated beams "meet" in the ER part of the linac.

beams) in the opposite directions. At the high-energy end of the linac the beams will be separated by the last dipole magnet of the return arc. Similarly, at the low energy end the most downstream magnet of the bunch compressor, BC II, will separate both beams appropriately and will also direct the returning beam to the dump. In all other nine locations, beams can be separated via simple bypasses (Fig. 10) based on four dipole magnets. The chicane parameters (for $10 \mathrm{~mm}$ horizontal displacement at $15.5 \mathrm{GeV}$ ) are listed in Table II. The magnetic field of the bypass scales linearly with the beam momentum. The total length of a bypass chicane with additional space needed for installation between cryostats is about $6 \mathrm{~m}$. All chicanes together extend the linac by $54 \mathrm{~m}$. The linac layout will allow for operation with beams for which $t_{b}$ is a multiple of $1 \mu \mathrm{s}$. The distance between midplanes of two bypasses (zone) is $150 \mathrm{~m}$. If experiments require an increase in the bunch repetition frequency, one can simply double the number of separation points and use $75 \mathrm{~m}$ long zones. This will allow a further increase in the flexibility in the beam time structure and operation of beams for which $t_{b}$ is a multiple of $0.5 \mu \mathrm{s}$. In this case the total linac will

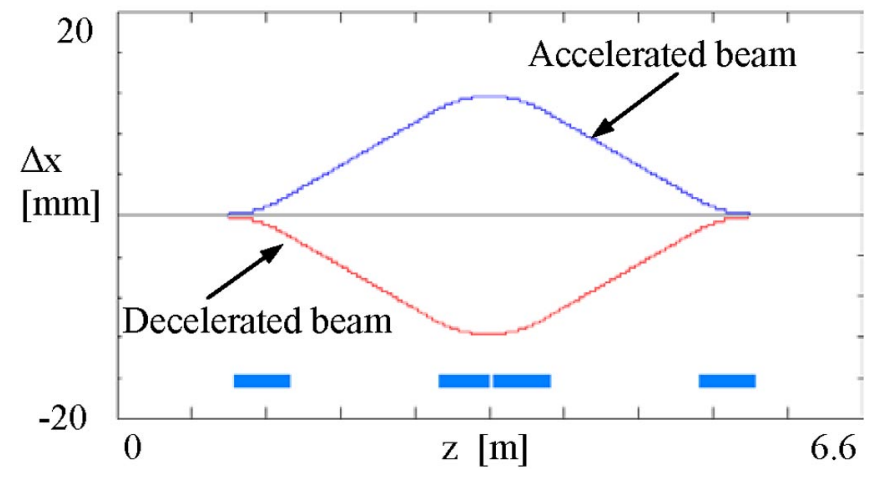

FIG. 10. (Color) Horizontal displacement of both beams in the bypass chicane (four identical dipole magnets - marked as blue bars). 
TABLE II. Parameters of the bypass chicane for $15.5 \mathrm{GeV}$.

\begin{tabular}{lcc}
\hline \hline \multicolumn{1}{c}{ Parameter } & Units & \\
\hline Beam energy & $\mathrm{GeV}$ & 15.5 \\
Beam separation & $\mathrm{mm}$ & 20 \\
Chicane length & $\mathrm{mm}$ & 4630 \\
Dipole magnet length & $\mathrm{mm}$ & 500 \\
$\theta_{\text {bend }}$ & $\circ$ & 0.366 \\
$\rho$ & $\mathrm{m}$ & 78 \\
$B$ & $\mathrm{~T}$ & 0.66 \\
Synchrotron radiation losses & $\mathrm{keV}$ & 265 \\
$D_{\text {max }}$ & $\mathrm{mm}$ & 10 \\
$M_{56}$ & $\mathrm{~mm}$ & -0.13 \\
\hline \hline
\end{tabular}

increase by $120 \mathrm{~m}$. We will continue with $150 \mathrm{~m}$ long zones, as shown schematically in Fig. 9.

\section{E. Short trains of bunches}

The proposed bypass chicanes are roughly $20 \lambda$ long (distance between the entrance and the exit dipole). They can accommodate trains of up to 20 bunches with the smallest possible spacing of one wavelength $(\lambda)$. These short trains of the accelerated and decelerated beams will still stay separated in the ER part of the linac. The highest repetition frequency of the short trains is the same as the highest repetition frequency of the nominal beam. ${ }^{5}$

\section{F. Long train}

The circumference of the return arc is about $1300 \mathrm{~m}$. It can accommodate (together with the $\sim 700 \mathrm{~m}$ long straight beam line needed for the collimation system, photon diagnostics, and undulators) a $6.7 \mu$ s long train of bunches. One can notice in passing that such a train, after full acceleration and subsequent passage through all the insertion devices, will return back to the linac without encountering any beam-beam interaction. The maximum number of bunches (for bunch spacing of one wavelength) is $\sim 8700$. To keep the beam energy constant along the bunch train one needs to adjust the phase of consecutive bunches to compensate for the cavity gradient drop (which depends on the total charge of the train). The required phase corrections are small if the total charge (in the train) is of the same order as the charge of the nominal beam passing through the linac within train duration $(\sim 7 \mathrm{nC})$. In this case, the energy difference between the first and the last bunch in the train will be $31 \mathrm{MeV}$ (for beam energy of $17 \mathrm{GeV}$ ). This can be compensated to $8 \mathrm{MeV}$ by a proper phasing of both linac parts (phase correction over the whole train duration is $3^{\circ}$ ). Then the next train can be generated and may enter the linac when the last bunch of the previous train reaches the beam dump; the trains can

\footnotetext{
${ }^{5} \mathrm{~A}$ different type of low charge and high frequency rf gun will be needed to generate above beam structure.
}

repeat every $24 \mu \mathrm{s}$. There is still more flexibility in the time structure of the beam within the long train, allowing accommodation of the additional requirements of FEL experiments. (See footnote ${ }^{5}$ )

\section{G. Building blocks}

The first part of the linac, up to $0.5 \mathrm{GeV}$ as shown in Fig. 7, will operate in a standard accelerating mode (no ER) and at constant gradient. Thirty-two 9-cell TTF-like cavities operating nominally at $15.2 \mathrm{MV} / \mathrm{m}$ are needed to reach that energy. On average, $15.6 \mathrm{~kW}$ of the rf power per cavity will be transferred via a fundamental power coupler (FPC) to the nominal $1 \mathrm{~mA}$ beam. This power level is well below the peak power specified and measured for pulsed operation of the FPC $(240 \mathrm{~kW})$; therefore, no multipacting or other voltage induced phenomena in the FPC are expected. The only concern is a possible heating of the FPC due to the larger duty factor. This will require further reconsideration of thermal effects in couplers and it may call for cooling improvements in the present design, if necessary. A layout of the ER part of the linac can be configured utilizing the so-called superstructures (SST) "chains" of weakly coupled multicell superconducting structures, first proposed in 1999 [15]. In 2002, the first beam test of two cold prototypes was conducted in the TTF linac at DESY, confirming their expected rf properties [16]. The layout allows for significantly reduced number of FPCs in an accelerator (a whole chain is "fed" by only one FPC) and it simultaneously provides excellent damping of HOMs. rf power transferred to the beam from external sources will be relatively small in the ER part. One can save substantially on the capital cost by making the accelerating structures long (with many cells). Here, we propose to use the $2 \times 9$-cell superstructures (Fig. 11) in the ER part of the linac. This SST is also proposed as an alternative layout in the TESLA Technical Design Report [6] for the $800 \mathrm{GeV}$ upgrade of the collider. To synchronize both beams with accelerating (decelerating) fields in the linac, the distance between SSTs in the cryomodules is fixed to one $\lambda$. Each cryomodule, e.g., based on the DESY design, will house four SSTs, one quadrupole, a steering coil package, and a beam position monitor. The cryomodule is $48 \lambda$ long and the distance between cryomodules is

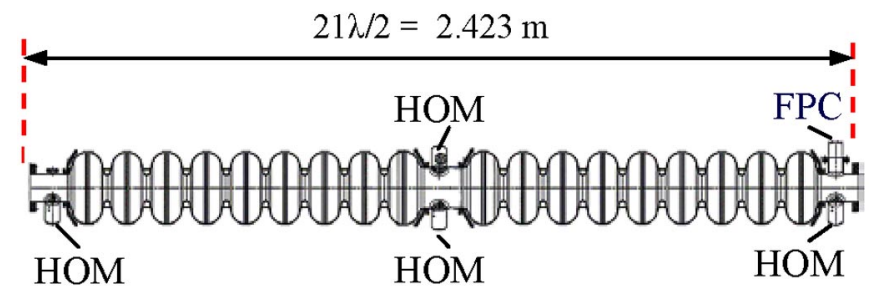

FIG. 11. (Color) $2 \times 9$-cell SST with additional space of $2 \lambda / 2$ at both sides. Note that these 18 cells are equipped with only one FPC and four HOM couplers. 


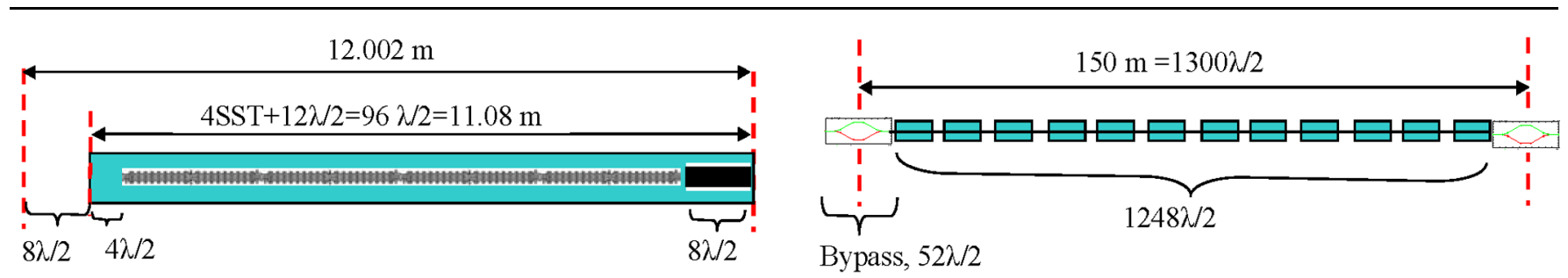

FIG. 12. (Color) Cryomodule housing four SSTs. Space for focusing elements (quads) and beam position monitor is marked

FIG. 13. (Color) A 12 cryomodule rf zone. as a black bar on the right.

TABLE III. Estimated normalized energy loss (in \%) for the nominal beam at various energies.

\begin{tabular}{lllc}
\hline \hline \multicolumn{1}{c}{ Final energy } & $9(\mathrm{GeV})$ & $17(\mathrm{GeV})$ & $25(\mathrm{GeV})$ \\
\hline Total radiation in all IDs & -2.5 & -2.5 & -2.5 \\
Return arc SR & -0.06 & -0.43 & -1.38 \\
All chicanes SR (both beams) & -0.001 & -0.008 & -0.02 \\
HOMs (both beams) & -0.33 & -0.18 & -0.12 \\
Energy gain in the first part & 5.6 & 2.9 & 2.0 \\
Maximum ER in the second part & 100 & 99.8 & 98.0 \\
\hline \hline
\end{tabular}

$4 \lambda$. The whole unit is shown in Fig. 12. A $150 \mathrm{~m}$ long zone accommodates 12 cryomodules and one bypass chicane as illustrated in Fig. 13. The overall fill factor of the zone is $67 \%$. The net energy gain (assuming a gradient of $15 \mathrm{MV} / \mathrm{m}$ ) is $31.25 \mathrm{MeV}, 125 \mathrm{MeV}$, and $1.5 \mathrm{GeV}$ in one SST, one cryomodule, and one zone, respectively.

\section{H. Energy recovery}

Percentages of energy losses for the entire facility (assuming the nominal beam structure) are collected in Table III. The net radiation in all photon devices is assumed to be in the range of $2.5 \%$ of the total beam energy. Synchrotron radiation (SR) in the return arc, when arc radius is $200 \mathrm{~m}$, stays below $1.4 \%$. The synchrotron radiation in the bypass chicanes is negligibly small (even at $25 \mathrm{GeV}$ ). The accelerated beam is composed of very short bunches, which increases energy deposition in the parasitic longitudinal modes of cavities. The low frequency part of this power will be transferred to external loads by HOM couplers. The higher frequency part will be absorbed by the beam line HOM absorbers.

The longitudinal loss factor $k_{\|}$, which scales approximately with the bunch length as $\sim 1 / \sqrt{\sigma_{z}}$, serves as a measure of energy deposition. For an 18-cell SST, assembled in a multistructure cryomodule, the average $k_{\|}$ is $40 \mathrm{~V} / \mathrm{pC}$ (for $\sigma_{z}=0.025 \mathrm{~mm}$ ) [17]. The decelerated beam will also deposit energy into the HOMs; however, its power loss can be significantly reduced by lengthening of the bunches. Appropriate optics choices in the return arc architecture (e.g., adjustable momentum compaction by design) will facilitate the above requirements (see Sec. II L on the arc design). A substantial fraction of the beam energy can be recovered in the second part of the linac (see last row of Table III). We assume (with a reasonable safety margin) that at least $96 \%$ of the beam energy can still be recovered (in the second part of the linac), even for $25 \mathrm{GeV}$ operation. ${ }^{6}$

\section{Microphonics}

Suppression of frequency modulation caused by mechanical cavity vibration (microphonics) is the second crucial technical challenge (following the $\mathrm{cw}$ low emittance rf-gun design). Figure 14 shows the rf power needed to operate an 18-cell SST for different final beam energies versus peak-to-peak frequency modulation. According to the estimate presented in [18], $5 \mathrm{~kW}$ of $\mathrm{rf}$ power will be sufficient to compensate both the "residual" beam loading as well as the microphonics. The facility can stably operate up to $20 \mathrm{GeV}$, if microphonics does not exceed $16 \mathrm{~Hz}$. Operation at higher energies will require additional $\mathrm{rf}$ power, or better suppression of mechanical vibrations. Measurements at FZ-Rossendorf on TTF cavities [19] showed that proper cryomodule construction may lead to an effective reduction of the frequency modulation to $10 \mathrm{~Hz}$ ( $2 \mathrm{~Hz} \mathrm{rms}$ ), as compared to $24 \mathrm{~Hz}$ of the original TTF cryomodule design [20]. In addition, one can apply an active vibration compensation by means of piezoelectric actuators similar to the Lorentz force detuning compensation in superconducting cavities [21].

\section{J. Linac parameters}

Table IV summarizes parameters of the first part of the linac (including the cryomodule of the injector) operating

\footnotetext{
${ }^{6}$ Deceleration off crest might be needed to compensate for the energy spread.
} 


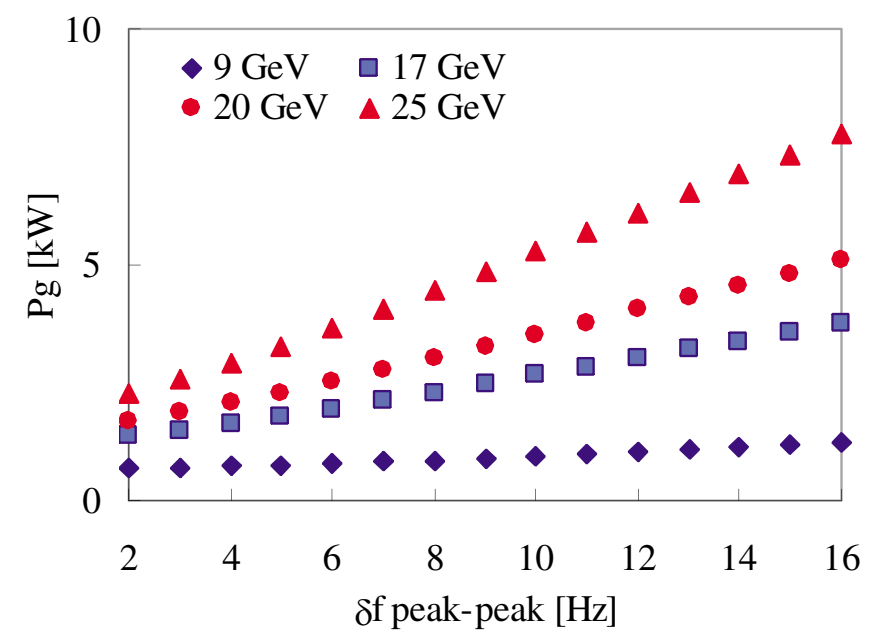

FIG. 14. (Color) Generator power needed to operate 18-cell SST for four final beam energies versus peak-to-peak microphonics.

for the nominal beam structure at constant gradient. As was mentioned before, this part should operate in the normal accelerating mode (without ER). Parameters of the ER linac are summarized in Table V. The maximum tolerable peak-to-peak microphonics has been computed assuming a maximum of $5 \mathrm{~kW} / \mathrm{SST}$ of available generator power. The maximum beam on-time was computed assuming $5.6 \mathrm{~kW}$ (at $2 \mathrm{~K}$ cryogenic budget) in the ER linac. The budget covers static heat and dynamic losses coming from the fundamental mode as well as from the HOMs excited by the nominal accelerated and decelerated beams. This estimate is based on the cryogenic budget breakdown given in TESLA [6]. The average beam power at the dump is quite moderate. There is no necessity for a bigger than $1 \mathrm{MW}$ dump (CEBAF size) as long as the total stored charge in the facility does not exceed the one for the nominal beam and the response time of an interlock system is shorter than $100 \mathrm{~ms}$.

\section{K. rf system for the ER part of the linac}

Without ER, 9 to $20 \mathrm{MW}$ of $\mathrm{rf}$ power would be needed to operate this part of the linac with nominal beam in the final

TABLE IV. Parameters of the first part of the linac.

\begin{tabular}{lcc}
\hline \hline \multicolumn{1}{c}{ Parameter } & Units & \\
\hline Energy at the exit & $\mathrm{MeV}$ & 500 \\
Input energy & $\mathrm{MeV}$ & 6.5 \\
$E_{\mathrm{acc}}$ & $\mathrm{MV} / \mathrm{m}$ & $15.2(13.6)^{\mathrm{a}}$ \\
Number of 9-cell structures & $\ldots$ & 32 \\
$f$ of accelerating mode & $\mathrm{MHz}$ & 1300 \\
$(R / Q)$ & $\Omega$ & 1038 \\
$Q_{o}$ & $10^{10}$ & 1.7 \\
$Q_{\text {ext }}$ & $10^{7}$ & $1.6(1.5)^{\mathrm{a}}$ \\
Total cryogenic load at $2 \mathrm{~K}$ & $\mathrm{~kW}$ & 0.5 \\
rf power & $\mathrm{MW}$ & 0.494 \\
\hline \hline
\end{tabular}

${ }^{\mathrm{a}}$ Gradient and $Q_{\mathrm{ext}}$ for the injector cryomodule. energy range from 9 to $20 \mathrm{GeV}$. ER operation significantly reduces this power (to less than $3 \mathrm{MW}$ ). Although this level of rf power can easily be supplied by three $1.2 \mathrm{MW}$ klystrons, we do not see this as a practical solution, mainly because of the complicated and expensive distribution system (176 SSTs/klystron) and technical difficulties in controlling amplitudes and phases in all the cavities. One can think of a much more attractive rf system ${ }^{7}$ based on $20-25 \mathrm{~kW}$ inductive output tubes (IOT). One IOT can be directly attached to one cryomodule housing four SSTs. The gain of these devices is $20-23 \mathrm{~dB}$, so that a $130-$ $200 \mathrm{~W}$ amplifier will be needed to drive one IOT. The IOT efficiency can be as high as $72 \%$. The other, probably more expensive option, is to use $20 \mathrm{~kW}$ klystrons of $50 \%$ efficiency. The choice of the rf system is still an open question, but one may expect substantial capital cost saving, compared to the rf system of a non-ER XFEL.

\section{Return teardrop ring}

The return teardrop ring (Fig. 7) is based on a periodic triplet focusing structure, which is a smooth continuation of linac FODO focusing. The ring is composed of 120 identical "inward bending" periodic cells and 40 "outward bending" cells closing a $180^{\circ}$ ring.

The basic cell architecture (two dipoles and a focusing triplet) is shown in Fig. 15 (beta functions and dispersion). The two kinds of cells differ only in the sign of the horizontal dispersion (mirror reflection). To make the ring achromatic the first/last two cells are differentthey have one dipole (right/left) removed in order to zero the horizontal dispersion and its derivative outside the ring. Similarly a three-cell-transition insert is placed at the junction point between the "inward-outward" parts of the ring. The first cell has the "right" dipole removed, in the second one both dipoles are missing, and the third cell has the "left" dipole removed in order to "flip" the sign of the horizontal dispersion, while maintaining the periodicity of the Twiss functions. The ring optics is illustrated in Fig. 16. The betatron phase advance per cell is chosen to be $90^{\circ}$. That is preferable from the point of view of chromatic effects compensation. The period length is slightly shorter (about $8 \mathrm{~m}$ ) than for the linacs to achieve the desired small value of $M_{56}$ (about $64 \mathrm{~cm}$ ). Triplet focusing has a few advantages over FODO focusing structure. First, it has larger separation between quads, which significantly simplifies compact ring design. Furthermore, it allows simple and smooth beam envelope matching from linac to return ring, which is very important for beams with the anticipated energy spread. Finally, triplet focusing has half the chromaticity of the vertical beam envelope of the FODO focusing and it requires chromatic corrections only for horizontal degree of freedom.

\footnotetext{
${ }^{7}$ This section is based on an unpublished study at BNL.
} 
TABLE V. Parameters of the ER part of the linac.

\begin{tabular}{|c|c|c|c|c|c|}
\hline Parameter & Units & & & & \\
\hline Input beam energy & $\mathrm{GeV}$ & \multicolumn{4}{|c|}{0.5} \\
\hline Type of SST & $\cdots$ & \multicolumn{4}{|c|}{$2 \times 9$ cell } \\
\hline$f$ of $\pi-0$ mode & $\mathrm{MHz}$ & \multicolumn{4}{|c|}{1300} \\
\hline$(R / Q)$ & $\Omega$ & \multicolumn{4}{|c|}{1970} \\
\hline Number of SSTs & $\cdots$ & \multicolumn{4}{|c|}{528} \\
\hline Number of cryomodules & $\cdots$ & \multicolumn{4}{|c|}{132} \\
\hline Number of zones & $\cdots$ & \multicolumn{4}{|c|}{11} \\
\hline Number of FPCs & $\cdots$ & \multicolumn{4}{|c|}{528} \\
\hline Crygenic budget at two K & $\mathrm{kW}$ & \multicolumn{4}{|c|}{$8^{\mathrm{a}}$} \\
\hline rf budget & $\mathrm{kW}$ & \multicolumn{4}{|c|}{2640} \\
\hline ER ratio & $\cdots$ & \multicolumn{4}{|c|}{0.96} \\
\hline Energy gain & $\mathrm{GeV}$ & 8.5 & 16.5 & 19.5 & 24.5 \\
\hline$E_{\mathrm{acc}}$ & $\mathrm{MV} / \mathrm{m}$ & 7.7 & 15.0 & 17.8 & 22.3 \\
\hline$Q_{o}$ & $10^{10}$ & 1.8 & 1.7 & 1.6 & 1.3 \\
\hline$Q_{\text {ext }}$ & $10^{7}$ & 7.5 & 7.9 & 8.0 & 14.0 \\
\hline $\operatorname{Max} \delta f$ & $\mathrm{~Hz}$ & $>18$ & 18 & 15 & 9 \\
\hline \multicolumn{6}{|l|}{$p-p$} \\
\hline Beam on-time $/ \mathrm{s}$ & $\mathrm{ms}$ & 1000 & 308 & 206 & 88 \\
\hline Number of bunches/s & $10^{6}$ & 1 & 0.308 & 0.206 & 0.088 \\
\hline Avg. brilliance ${ }^{b}$ & $10^{25}$ & 1.64 & 6.4 & 8.2 & 8.6 \\
\hline Peak beam power & MW & 9 & 17 & 20 & 25 \\
\hline$\langle$ Power at dump $\rangle$ & MW & 0.84 & 0.36 & 0.27 & 0.13 \\
\hline
\end{tabular}

${ }^{\mathrm{a}} 8 \mathrm{~kW}$ result from the $5.6 \mathrm{~kW}$ cryogenic load $+40 \%$ overhead.

${ }^{\mathrm{b}}$ Units: photon $/ \mathrm{s} / \mathrm{mm}^{2} / \mathrm{mrd}^{2} / 0.1 \%$ bandwidth.

Another crucial beam transport issue is to maintain manageable beam sizes. This calls for short cells and for putting stringent limits on dispersion and beta functions (beam envelope).
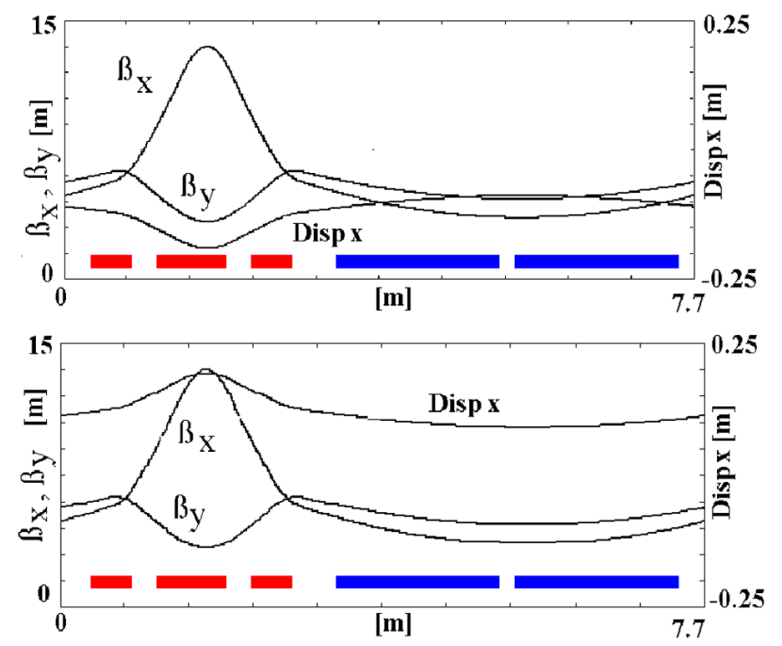

FIG. 15. (Color) Twiss functions and dispersion of the periodic cells in the return ring. Inward cells (upper diagram), outward cell (lower diagram). Bars illustrate magnets (red short quads, long blue dipoles).

\section{MOTIVATION}

\section{A. XFEL operation}

One of the attractive aspects of cw operation is the possibility of providing large spacing between FEL pulses, while keeping the total repetition rate high. There seems to be unanimous agreement that for a pump-probe experiment, synchronization of the FEL facility with a conventional laser is of paramount importance. This means that a $200 \mathrm{~ns}$ spacing would be too close, while a $1 \mu$ s spacing is sufficient for most pump-probe experiments.

Furthermore, it is the spacing that allows multiplexing bunches into multiple beam lines, resulting in a larger spacing in the individual beam lines. Let us assume ten beam lines; each beam line may then have a laser synchronized to the beam, running at $100 \mathrm{kHz}$. For some experiments $10 \mathrm{kHz}$ may be even better.

There are two main scientific reasons for a higher brightness light source such as this one:

(i) "Round beams," in which the horizontal emittance is approximately the same as the vertical. This gives between 2 and 3 orders of magnitude gain over storage rings and allows, for example, smaller protein crystals to be studied with high resolution. It also minimizes effects due to the mosaic spread of larger crystals.

(ii) Shorter pulses, typically subpicosecond instead of several tens of picoseconds. These allow dynamical studies 

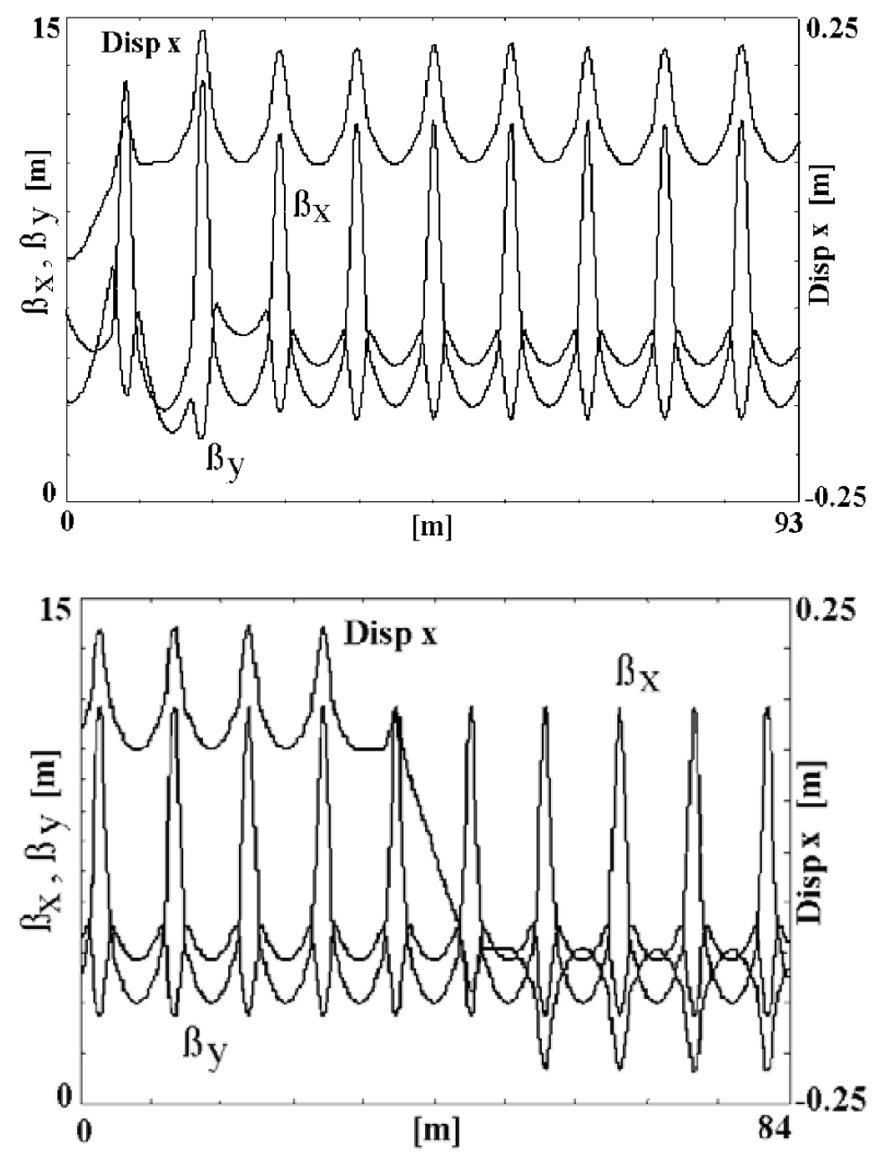

FIG. 16. Dispersion and $\beta$ functions of a regular inward sector of the return ring (upper diagram) and the "transition" insert between the inward and outward cells (lower diagram).

on time scales relevant to chemical bond-breaking (50 fs) and electron-phonon interactions.

In combination these two advantages allow out-ofequilibrium pump-probe dynamical experiments [22-26] in which the high repetition rates allow significant gains due to averaging in systems in which recovery occurs between the pulses. In these latter experiments, the small round beams also closely match those of the excitation lasers. It should be noted that the LCLS and the DESY XFEL are examples of facilities that lie at an important but extreme end of a pulse repetition frequency spectrum, while facilities such as those proposed here offer a capability that would cover a different and possibly broader base.

\section{B. Nuclear physics operation}

Another versatile feature of the proposed XFEL complex is that the driving linac itself, operating in the normal accelerating mode (cw without ER) is capable of delivering electron beams with energies as high as $20 \mathrm{GeV}$ and currents up to $50 \mu \mathrm{A}-$ a very attractive facility for nuclear physics studies. This scenario assumes a $5 \mathrm{~kW}$ budget of $\mathrm{rf}$ power per SST, compensating both the beam loading and microphonics $(<14 \mathrm{~Hz})$, as illustrated in Fig. 17.

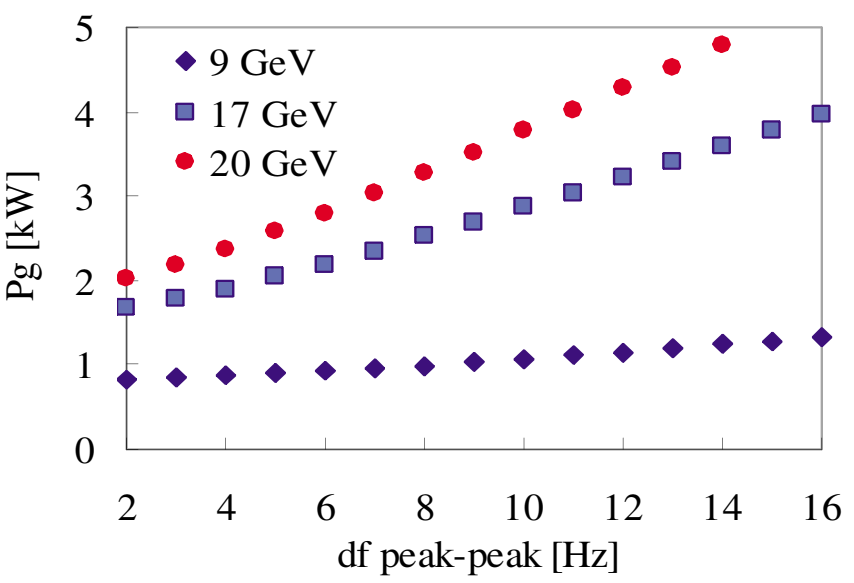

FIG. 17. (Color) rf power needed to operate SST without ER versus microphonics at three different final beam energies and beam current of $50 \mu \mathrm{A}$.

\section{R\&D PROGRAMS}

The scheme presented for a future energy recovery, $\mathrm{cW}$ XFEL gives a preliminary answer to our first question. It is a good starting point-no "show stoppers" have been found so far. Thorough assessment of collective effects will follow. However, it should undergo further optimization driven by experimental needs as determined by the user community. Obviously a different layout, based on the injection of the decelerated beam from the low energy side, can also be considered as an alternative option, which will be less demanding on the cavities spacing tolerance. It would require an additional transfer line and two return teardrop rings at each end of the ER part, if the returning beam line is installed in the same tunnel as the accelerator. This seems to make the "two arcs" option more expensive than the one presented. Additionally, the optics and focusing scheme are more challenging but not impossible, as it was recently demonstrated by the CEBAF energy recovery experiment.

\section{A. rf gun}

The answer to the second question is more complex. This discussion confirms that present $\mathrm{R} \& \mathrm{D}$ programs leading to $\mathrm{cw}$ low emittance rf-gun design should be continued to take full advantage of a cw facility. The increasing quantum efficiency of $\mathrm{Nb}$ (the BNL approach) and/or testing of photoemission from other superconductors $(\mathrm{Pb})$ should be investigated. Furthermore, the use of shorter wavelength lasers $(\lambda<248 \mathrm{~nm})$ to illuminate the photoemitting area should be tested with other superconducting materials.

\section{B. Microphonics}

The second R\&D topic-microphonics-is directly related to capital and operation costs. Better suppression of microphonics lowers the rf system cost, since the required rf budget scales almost linearly with the microphonics amplitude (see Fig. 14). There has not been 
much experience with operating multicell superconducting cavities when assembled in a multistructure cryomodule with $Q_{\text {load }}$ as high as $7 \times 10^{7}-2 \times 10^{8}$. However, a simple estimate shows that $5 \mathrm{~kW} / \mathrm{SST}$ of $\mathrm{rf}$ power would be sufficient to operate the ER part of the linac, at least up to $20 \mathrm{GeV}$. Therefore, there is a significant need for an experimental demonstration of the phase and the amplitude stability control for such high $Q_{\text {load }}$. In the very near future, a test of the $12 \mathrm{GeV}$ CEBAF upgrade cavities will be performed at JLab. These cavities will be operated $\mathrm{cw}$ with $Q_{\text {load }} 1.5 \times 10^{7}$, according to the upgrade specification.

\section{Cavities}

Routinely achievable accelerating gradients in superconducting cavities already exceed the gradients needed to operate the cw XFEL facility (presented here) even beyond the final beam energies of both large facilities at DESY and at SLAC. An important improvement in cavity performance is enhancement of intrinsic $Q$ at the operating gradient. In addition, novel production and preparation methods should ensure its reproducibility and repeatability. Similar to the suppression of microphonics, higher $Q$ will lower the total cost of the facility (cryoplant costs) and/or will allow for longer beam on-time in semi-cw operation.

As was mentioned in this study, Nb prototypes of SST $(2 \times 7$ cells) were tested in 2002 with a beam at DESY. Damping of HOM was very good and the TESLA collider specification has been met with a large margin. Damping in $2 \times 9$-cell SST was tested only on Cu models. This bench measurement showed that damping is sufficient to operate the collider with its nominal current of $9 \mathrm{~mA}$. The nominal current of the single arc option is only $1 \mathrm{~mA}$ and the damping specification will allow for much higher $Q_{\text {ext }}$, scaled $\sim(9 \mathrm{~mA}) /(2 \mathrm{~mA})$. Unlike other presently proposed ER facilities, this one will have no problem with HOM suppression.

\section{Energy recovery}

Future energy recovery experiments at CEBAF at higher than $1 \mathrm{GeV}$ energy will be very helpful to confirm that decelerated beam quality can be preserved for higher (than 50) full to injection energy ratios. This is of more importance for the two arcs option since the single arc option does not "suffer" from compromised focusing for very different energy beams.

We notice in passing that all the R\&D programs essential to demonstrating feasibility of the cw single arc XFEL facility are already in progress in many leading laboratories, e.g., BNL, JLab, and DESY.

\section{SUMMARY AND FINAL REMARKS}

\section{A. Capital and operation costs}

Neither capital nor operation costs are expected to be lower than those of the DESY XFEL presented in [2].
Significant savings in the capital cost of the rf system (sources, power supplies, and distribution) and cost savings resulting from the SST layout may cover part of the additional costs due to $16 \%$ more cavities, larger cryoplant, and the return teardrop arc. All additional costs are difficult to estimate, at the moment, since they strongly depend on progress in $R \& D$ programs over the next few years. Operation of a future $\mathrm{cw}$ facility will require about 9 MW power and operation costs will be higher than for the pulsed DESY XFEL facility (3.5 MW). This cost estimate also depends on the progress of future $R \& D$ programs.

\section{B. Average brilliance}

These additional costs may be justified by the increased performance and flexibility of the proposed facility. The cw XFEL will "boost" the average brilliance by a factor of 25 at $9 \mathrm{GeV}$, or a factor of 5 at $20 \mathrm{GeV}$ (compared to the average brilliance of the pulsed version). Furthermore, it will extend the energy range beyond $20 \mathrm{GeV}$ without any additional cost.

\section{ACKNOWLEDGMENTS}

The authors thank C. Rode, M. Tiefenback, and G. Wu for providing useful information included in this paper and for helpful discussions. This work is supported in part by the U.S. DOE under Contract No. DE-AC05-84ER40150.

[1] Linac Coherent Light Source-CDR, Report No. SLAC-R593, 2002.

[2] TESLA Technical Design Report (Supplement), DESY No. TESLA-FEL Note 2002-09, edited by R. Brinkmann et al., 2002.

[3] S. A. Bogacz et al., in Proceedings of the Particle Accelerator Conference (PAC03), Portland, OR, 2003 (IEEE, Piscataway, NJ, 2003).

[4] H. Weise, in Proceedings of the Particle Accelerator Conference (PAC03), Portland, OR, 2003 (Ref. [3]).

[5] P. Kneisel et al., in Proceedings of the 11th Workshop on RF-Superconductivity (SRF2003), Lübeck, Germany 2003 (unpublished).

[6] TESLA Technical Design Report, DESY No. TESLA Note 2001-23, edited by R. Brinkmann et al., 2001.

[7] J. Teichert, in Proceedings of the 11th Workshop on RFSuperconductivity (SRF2003), Lübeck, Germany 2003 (Ref. [5]).

[8] T. Srinivasan-Rao et al., in Proceedings of the Particle Accelerator Conference (PAC03), Portland, OR, 2003 (Ref. [3])

[9] P. Kneisel, JLab-TN (to be published).

[10] T. Schultheiss et al., in Proceedings of the Particle Accelerator Conference (PAC01), Chicago, IL, 2001 IEEE, Piscataway, NJ, 2001. 
[11] M. Ferrario, Nucl. Instrum. Methods Phys. Res., Sect. A 472, 303 (2001).

[12] L. Serafini et al., Phys. Rev. E 55, 75651997.

[13] M. Ferrario et al., "An Ultra-high Brightness, High Duty Factor, Superconducting RF Photoinjector" (to be published).

[14] D. Douglas, Report No. JLab-TN-98-040, 1998.

[15] J. Sekutowicz et al., Phys. Rev. ST Accel. Beams 2, 062001 (1999).

[16] J. Sekutowicz et al., in Proceedings of the Particle Accelerator Conference (PAC03), Portland, OR, 2003 (Ref. [3]).

[17] R. Brinkmann et al., TESLA Report No. 2000-07, DESY, 2000.
[18] L. Merminga and J. Delayen, Report No. JLab TN-96-022, 1996.

[19] A. Büchner et al., in Proceedings of the Particle Accelerator Conference (PAC03), Portland, OR, 2003 (Ref. [3]).

[20] M. Liepe, Report No. DESY-Thesis-2001-045, 2001.

[21] M. Liepe et al., in Proceedings of the Particle Accelerator Conference (PAC01), Chicago, IL, 2001 (Ref. [10]).

[22] R. Neutze et al., Phys. Rev. Lett. 87, 195508 (2001).

[23] S. Techert et al., Phys. Rev. Lett. 86, 2030 (2001).

[24] G. L. Carr et al., Phys. Rev. Lett. 85, 3001 (2000).

[25] Z. Ren et al., Biochemistry 40, 13788 (2001).

[26] V. Strejer et al., Biochemistry 40, 13802 (2001). 